Check for updates

Cite this: Chem. Sci., 2018, 9, 1323

\title{
One-step solvothermal synthesis of high-emissive amphiphilic carbon dots via rigidity derivation $\dagger$
}

\author{
Pei Zhao, (D) ${ }^{a}$ Xuping Li, ${ }^{a}$ Glib Baryshnikov, ${ }^{\text {bd }}$ Bin Wu, ${ }^{a}$ Hans Ågren, (D) be \\ Junji Zhang ${ }^{\mathrm{C}}{ }^{\mathrm{C}}$ and Liangliang Zhu (D) *a
}

In nanoscience, amphiphilic carbon dots (ACDs) are of great importance due to their excellent transferability for application in biological sensing, imaging and labelling. However, facile synthetic strategies are still limited, especially for obtaining high-emissive ACDs. Since the development of a highemissive feature is strongly desired for improving the practical resolution in vivo, here we report a chemical strategy that uses rigid molecules to straightforwardly construct amphiphilic carbon dots (ACDs) with high luminescence quantum yields (QYs). By using 1-[bis(dimethylamino)methylene]-1H1,2,3-triazolo[4,5-b]pyridinium 3-oxide hexafluorophosphate (HATU), a typical coplanar compound, as the only precursor, well-defined ACDs were prepared via a one-step solvothermal process which exhibited a superior QY of up to $29 \%$, largely superior to those prepared from precursors with less rigid structures. The effect can be mainly attributed to a significant suppression of the competition of nonradiative decay through rigidity derivation. Metal ionic doping during the synthesis resulted in a further improvement of the crystallinity and monodispersity of the materials, with retention of the high-emissive ability. This high-emissive photoluminescence behavior of the ACDs is accompanied with an excitationwavelength dependence, a high biocompatibility and a low toxicity, which together make the ACDs advantageous for application in multi-channel bioimaging.

Received 25th October 2017 Accepted 12th December 2017

DOI: $10.1039 / \mathrm{c} 7 \mathrm{sc0} 04607 \mathrm{c}$

rsc.li/chemical-science probably because the light-emitting process is readily quenched in a charge-separated form of the nanocrystals. Although the radiative decay process has been regulated by a variety of approaches in nanoscience (e.g. coating and post-modification, etc.) including in the field of CDs, ${ }^{7}$ it remains desirable, albeit a great challenge, to develop easy-to-handle synthetic strategies to directly access high-emissive ACDs.

Since high-emissive systems have been playing a significant role in the promotion of resolution and practicability in applied optoelectronics and in vivo, scientists are always seeking different ways to constantly improve material QYs. In the field of organic functional materials, the rigidity of molecular structures is essential to strengthen the radiative decay process by inhibiting competitive energy loss originating from molecular vibration and rotation. ${ }^{8}$ With these facts in mind, we try in this work to apply the concept of molecular rigidity to the synthesis of ACDs in anticipation that ligands or precursors with particular structures can provide effective rigidity derivation towards the formation of nanocrystal cores to facilitate the radiative emission process of the whole system. Moreover, an additional doping strategy to regulate the quality of the prepared nanomaterials is also considered. ${ }^{9}$

Herein we demonstrate the one-step solvothermal synthesis of ACDs from rigid molecular structures as the only precursors (see the illustration in Fig. 1). Generally, CDs can be synthesized by different "top-down"1,10 and "bottom-up" approaches. ${ }^{11}$ 


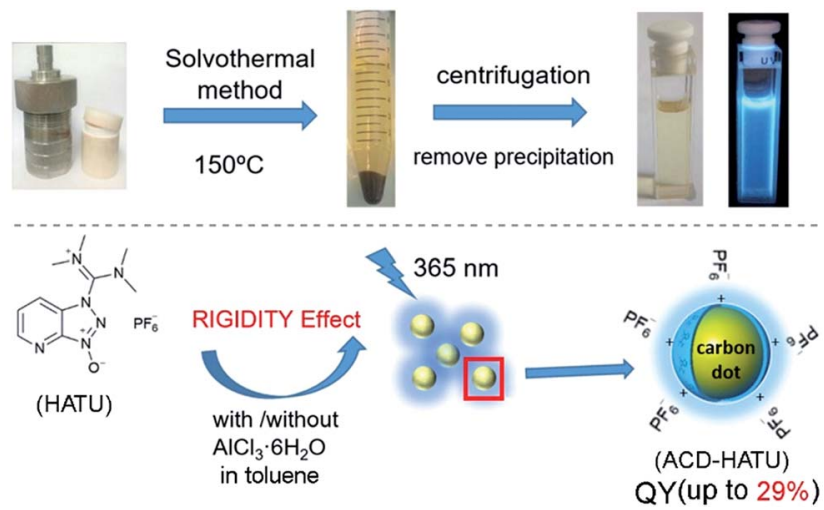

Fig. 1 Synthesis of ACDs from the derivation of rigid molecular structures by a one-step solvothermal method with the employment of a coplanar structure HATU as the only precursor. The synthesized ACDs show strong photoluminescence (QY up to $29 \%$ ) under UV light (excited at $365 \mathrm{~nm}$ ).

Solvothermal treatment is a cheap, environmentally friendly and nontoxic technique to produce CDs. ${ }^{11 e}$ In our work, a series of commercially-available heteroatomic compounds were used under relatively mild reaction conditions to conduct a rapid and facile synthesis to produce ACDs, and the results were analyzed from the perspective of rigidity derivation (see the detailed procedures in the Experimental section). We show that 1-[bis (dimethylamino)methylene]-1 $H$-1,2,3-triazolo[4,5- $b]$ pyridinium 3-oxide hexafluorophosphate (HATU) can serve as an ideal rigid precursor to form ACDs with high QYs, and that the crystallinity and monodispersity can be further improved by metal ionic doping. While the leading ACDs can also reveal an excitationwavelength dependent emission, our materials can achieve direct application in multi-channel cellular imaging, due to their additional superior biocompatibility and low biotoxicity. Such a preparation strategy might lead to a new concept for advancing fundamental nanoengineering to address high-tech light-emitting materials of different size scales.

\section{Experimental}

\section{Chemicals and reagents}

1-[Bis(dimethylamino)methylene]-1 $H$-1,2,3-triazolo[4,5- $b]$ pyridinium 3-oxide hexafluorophosphate $\left(\mathrm{C}_{10} \mathrm{H}_{15} \mathrm{OF}_{6} \mathrm{~N}_{6} \mathrm{P}, \mathrm{HATU}\right.$, $99.5 \%), \mathrm{AlCl}_{3} \cdot 6 \mathrm{H}_{2} \mathrm{O}$ (98\%), diisopropylamine (98\%), benzyltriethylammonium chloride (99\%), 1-benzylimidazole (98\%), toluene $(99.5 \%)$, ethanol $(99.7 \%)$, acetonitrile $(99 \%), \mathrm{N}, \mathrm{N}$ dimethylformamide (DMF, 99.5\%) and pyridine (99.5\%) were purchased from Energy Chemical, Vetec ${ }^{\mathrm{TM}}$, Alfa Aesar, Shanghai Dahe Chemical and Tansoole Company. All reagents were of analytical or reagent grades and used without further purification. Deionized water $(18.2 \mathrm{M} \Omega \mathrm{cm})$ was obtained from a F'DEER water purification system.

\section{Synthesis}

HATU (0.5 mmol) or other precursors (0.5 mmol benzyltriethylammonium chloride, $0.5 \mathrm{mmol}$ 1-benzylimidazole, $1 \mathrm{~mL}$ diisopropylamine) were added with stirring at room temperature to poly(tetrafluoroethylene) (Teflon) lined flasks $(50 \mathrm{~mL})$ containing $30 \mathrm{~mL}$ solvent. After 20 minutes of stirring they were transferred into a stainless steel autoclave and put in the oven. The autoclaves were heated up to $150{ }^{\circ} \mathrm{C}$, maintained at this temperature for 8 hours, and cooled to room temperature naturally in the oven. The yellowish solution and dark brown oily precipitate were separated by centrifugation. The doped materials were prepared in the same way only with $\mathrm{AlCl}_{3} \cdot 6 \mathrm{H}_{2} \mathrm{O}$ (1 mmol) added.

TEM. The (high-resolution) transmission electron microscopy, (HR)TEM, images were obtained on a JEOL JEM-2100F field emission electron microscope with $200 \mathrm{kV}$ field emission. The mother liquids were dissolved in $1 \mathrm{~mL}$ alcohol. The solutions were then dropped on an ultra-thin carbon membrane coated copper grid and the excess of the solution was removed with filter paper.

AFM. Atomic force microscopy (AFM) was conducted in tapping mode on a Bruker Multimode-8.

EDS. Energy dispersive X-ray spectroscopy (EDS) was carried out on an Ultra 55 field emission scanning electron microscope equipped with an EDS detector from Zeiss Company.

Raman. Raman spectroscopy was carried out using ACDs in aqueous droplets on the slide. The spectra were recorded on a Horiba Jobin Yvon XploRA spectrometer equipped with a $10 \times$ objective and a laser with a wavelength of $532 \mathrm{~nm}$. Data were collected between $850 \mathrm{~cm}^{-1}$ and $1700 \mathrm{~cm}^{-1}$ using a grating of 600 grooves per $\mathrm{mm}$. They were normalized using the LabSpec Version 5 software package.

FTIR. Fourier transform infrared (FTIR) spectroscopy was carried out with a Thermofisher Nicolet 6700 spectrometer using $\mathrm{KBr}$ pellets as the sample matrix in the wavenumber range of $400-4000 \mathrm{~cm}^{-1}$.

XPS. X-ray photoelectron spectroscopy (XPS) was performed on a PHI5300 using a magnesium K $\alpha$ source $(250 \mathrm{~W}, 14 \mathrm{kV})$.

UV-vis. The ultraviolet-visible (UV-vis) absorption spectra were measured in the region of $250-800 \mathrm{~nm}$ on a Shimadzu 1800 spectrophotometer.

PL. The photoluminescence (PL) excitation and emission spectra, and the lifetime and quantum yield, were recorded on a Shimadzu RF-5301, FLS 920 (Edinburgh Instruments) and a QM40 spectrofluorophotometer from Photo Technology International (PTI), respectively.

The quantum yields were determined using an integrating sphere. In this approach the absolute photoluminescence quantum yield is given by

$$
\begin{gathered}
\Phi=\left[E_{\text {in }}(\lambda)-(1-\alpha) E_{\text {out }}(\lambda)\right] /\left[X_{\text {empty }}(\lambda) \alpha\right] \\
\alpha=\left[X_{\text {out }}(\lambda)-X_{\text {in }}(\lambda)\right] / X_{\text {out }}(\lambda)
\end{gathered}
$$

In these equations, $E_{\text {in }}(\lambda)$ and $E_{\text {out }}(\lambda)$ are the integrated luminescence as a result of direct excitation of the sample and secondary excitation, respectively. The latter emission is due to reflected excitation light from the sphere walls hitting the sample. $X_{\text {empty }}(\lambda)$ is the integrated excitation profile with the 
empty sphere. $\alpha$ is the film absorptance. $X_{\text {in }}(\lambda)$ is the integrated excitation when the sample lies directly in the excitation path and $X_{\text {out }}(\lambda)$ is the integrated excitation when the excitation light first hits the sphere wall as previously explained. All of the spectra were recorded with the same excitation and emission monochromator bandpass. ${ }^{12}$

The relative quantum yields were determined in raw solvent using optically matching ethanol solutions of rhodamine $\mathrm{B}$ $\left(\Phi_{\mathrm{r}}=0.65\right)$ and quinine sulfate in $0.1 \mathrm{M} \mathrm{H}_{2} \mathrm{SO}_{4}\left(\Phi_{\mathrm{r}}=0.54\right)$ as references. The quantum yields were calculated using the below equation:

$$
\Phi_{\mathrm{f}}=\Phi_{\mathrm{r}}\left(A_{\mathrm{r}} F_{\mathrm{s}} / A_{\mathrm{s}} F_{\mathrm{r}}\right)\left(\eta_{\mathrm{s}}{ }^{2} / \eta_{\mathrm{r}}{ }^{2}\right)
$$

where $A_{\mathrm{s}}$ and $A_{\mathrm{r}}$ are the absorbance of the sample and reference solutions, respectively, at the same excitation wavelength, $F_{\mathrm{s}}$ and $F_{\mathrm{r}}$ are the corresponding relative integrated emission intensities, and $\eta$ is the refractive index of the solvent. ${ }^{13}$

\section{Cell labeling and cytotoxicity assay}

The cell viability was quantitatively determined by the Cell Counting Kit-8 (CCK-8) assays. Hela cells were obtained from the Cell Bank of the Chinese Academy of Science (Shanghai, China) and seeded onto a 96-well cell culture plate at a density of $1 \times 10^{4}$ cells per well in Dulbecco's modified Eagle medium (DMEM) containing $10 \% \mathrm{FBS}$ under $5 \% \mathrm{CO}_{2}$ at $37^{\circ} \mathrm{C}$. After the cells grew for $12 \mathrm{~h}$, the medium was changed into a new medium $(200 \mu \mathrm{L}$ per well) containing the carbon dots at 1000 , 500,100 and $50 \mu \mathrm{g} \mathrm{mL}^{-1}$. After the cells were incubated with the sample for $24 \mathrm{~h}$, the medium was replaced with $100 \mu \mathrm{L}$ of fresh medium. Subsequently, $10 \mu \mathrm{L}$ of CCK- 8 was added to each well and homogeneously mixed, followed by incubation at $37^{\circ} \mathrm{C}$ for $4 \mathrm{~h}$ in a $\mathrm{CO}_{2}$ incubator, and finally, $80 \mu \mathrm{L}$ of the solutions were put into a new 96-well plate. The optical density of each well at $450 \mathrm{~nm}$ was read using a microplate reader.

Hela cells were seeded in $35 \mathrm{~mm}$ plastic-bottomed dishes and grown in DMEM medium for $24 \mathrm{~h}$, then the cells were incubated in fresh medium containing $100 \mu \mathrm{g} \mathrm{mL}{ }^{-1}$ ACDs for another $4 \mathrm{~h}$. The cells were washed with a phosphate buffer saline (PBS) solution ( $\mathrm{pH}$ 7.4) three times and fixed with paraformaldehyde at $4{ }^{\circ} \mathrm{C}$ for $15 \mathrm{~min}$. The luminescence images of the cells were captured using a Nikon laser scanning confocal microscope $\mathrm{C}^{+}$with different channels.

\section{Computational details}

The structures of the precursor compounds and their isomers have been optimized at the density functional theory (DFT) level using the B3LYP ${ }^{14}$ hybrid exchange-correlation functional and the extended $6-311++\mathrm{G}(\mathrm{d}, \mathrm{p})$ basis set. ${ }^{15}$ The same method has been used to determine the true minimum of the total energy and corresponding vibrational frequencies. For the optimization procedure we have used the "tight" criterion for selfconsistence field (SCF) convergence starting from the different initial conformations of these compounds. All the detected isomers (conformers) with a true minimum on the potential energy surface (PES) are presented in Fig. 1 and S1. $\dagger$ Among them HATU and BTEAC were considered in the cationic form while counterions were omitted for clarity. The basic principle applied for the conformational analysis was a gradual rotation of the main functional groups along the corresponding $\sigma$-bonds with the subsequent optimization procedure. All DFT calculations have been performed using the GAUSSIAN16 (ref. 16) software. The QTAIM calculations ${ }^{17}$ have been realized within the AIMAll program package. ${ }^{\mathbf{1 8}}$

\section{Results and discussion}

In our work, ACDs were synthesized by solvothermal reactions from a series of precursors dissolved in toluene at $150{ }^{\circ} \mathrm{C}$ for $8 \mathrm{~h}$. The heteroatomic precursors include HATU, diisopropylamine (DIPA), benzyltriethylammonium chloride (BTEAC) and 1-benzylimidazole (BI), which were chosen due to their close molecular length and polarity to investigate the effect of molecular coplanarity and rigidity. All these precursors can produce ACDs with similar compositions and sizes under the same reaction conditions. However, by using these molecules for derivation, we observed that HATU leads to the highest luminescence QY (up to $29 \%$ in toluene) in the prepared ACDs (ACD-HATU) whereas the other precursors only yield ACDs (ACD-DIPA, ACDBTEAC and ACD-BI) with QYs below 2\% (0.6-1.7\%).

To gain the precise structural information of these precursors for comparison, computational analysis was firstly employed upon their optimized geometries. Since a coplanarity parameter can straightforwardly reflect the molecular rigidity, we measure the dihedral angle in these optimized geometries between the polar $\mathrm{C}-\mathrm{N}$ bond and the main bulk group, as shown in Fig. 2a. Due to the formation of an intermolecular hydrogen bond, the dihedral angle in HATU $\left(38^{\circ}\right)$ turned out to be smallest among these precursor structures. On the other hand, HATU bears more $\mathrm{sp}^{2}$ hybrid orbitals in its main aromatic skeleton than the other molecules. These results suggest that normally HATU will reveal the biggest conformational rigidity in comparison with the rest of this series of precursors. In contrast, the lack of coplanarity and the existence of different conformers (see Fig. $\mathrm{S} 1 \dagger$ ) readily make the other precursor molecules less rigid.

Furthermore, direct experimental evidence to confirm the rigidity derivation effect on the prepared ACDs can also be obtained from the Raman spectra. CDs generally exhibit a D-band $\left(\sim 1330 \mathrm{~cm}^{-1}\right)$ and a G-band $\left(\sim 1600 \mathrm{~cm}^{-1}\right) \cdot{ }^{19}$ In our case, a strong Raman peak, assigned to $\mathrm{C}-\mathrm{N}$ stretching vibrations $\left(V_{\mathrm{C}-}\right.$ $\mathrm{N}, \sim 1080 \mathrm{~cm}^{-1}$ ), was also observed. ${ }^{20}$ Since it would be understandable that the carbon core has a minimum sensitivity to the amphiphilic molecular structures at the rim of the nanosystems, the D-band and G-band were set as the internal standard to compare the normalized Raman spectra among these ACDs (Fig. 2b). Although these synthesized ACDs generally exhibit a similar size and number of atomic bonds, we find that the intensity of $V_{\mathrm{C}-\mathrm{N}}$ shows a great difference relative to the internal standard. The $V_{\mathrm{C}-\mathrm{N}}$ in ACD-HATU is the weakest one, indicating that the peripheral vibration behavior was significantly restricted. In terms of the above geometrical studies, we can conclude that the high coplanarity and strong rigidity effect 
(a)
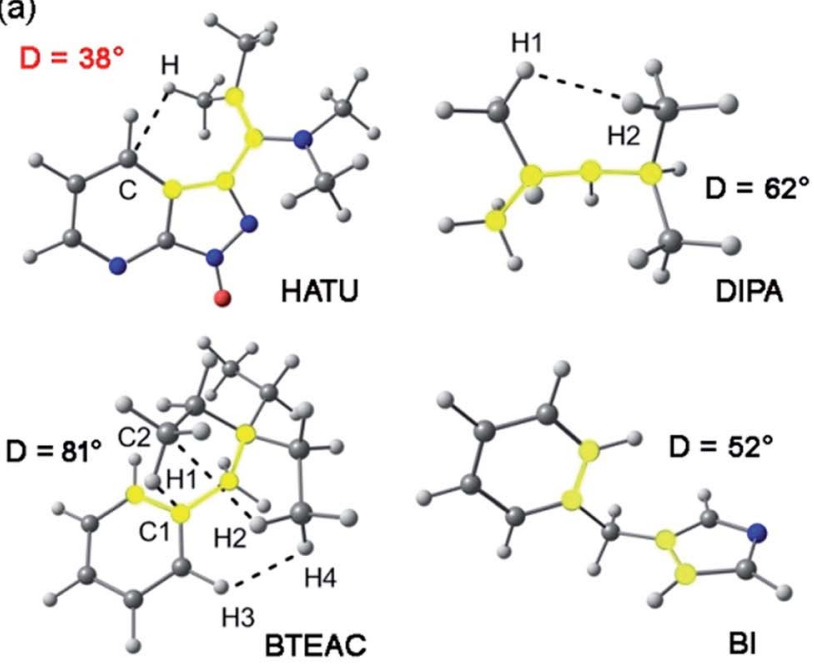

BI

(b)

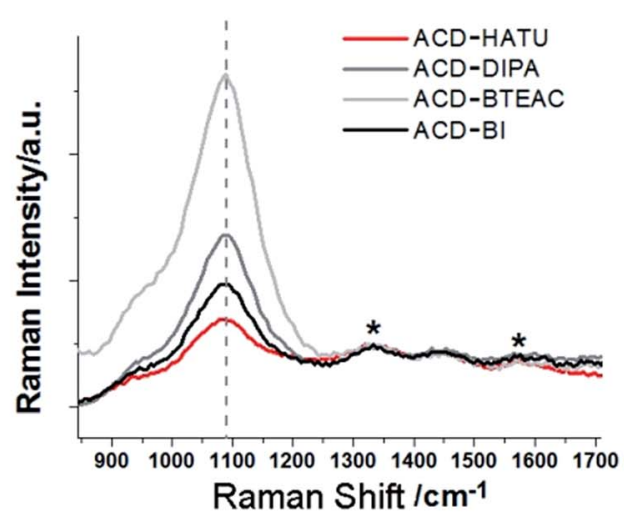

Fig. 2 Study of the molecular rigidity of the precursors: (a) coplanarity parameters in the optimized geometries obtained by B3LYP/6$311++G(d, p)$ of the precursor molecules. The dashed lines indicate the non-covalent interactions detected by AIM analysis of the electronic density distribution function. The atoms that compose the highlighted dihedral angles are shown in yellow. (b) Normalized Raman spectra of the ACDs in water. The internal standard signals are marked with "*".

of HATU greatly reduced molecular vibrations, so as to inhibit competitive energy loss and non-radiative decay and allow a remarkable enhancement of the QY of the whole nanocrystal. Since HATU itself cannot emit any luminescence (see the photographic comparison in Fig. S2 $\uparrow$ ), the as-prepared ACD with high QY can attract unique interest as a single nanoplatform. With these ideas in mind, we turn to explore the structural and photophysical features of ACD-HATU in detail.

Meanwhile, the FTIR spectrum was recorded to obtain more structural information of ACD-HATU, as shown in Fig. S3. $\dagger$ To further compare and investigate the role of HATU, we also replaced toluene with other solvents to monitor the environmental effect. The TEM and AFM images with associated height analysis (Fig. S4 and S5†) show that these dots have a spherical nanoshape with diameters in the range of $2-5 \mathrm{~nm}$. Fig. 3 a shows HRTEM images of ACD-HATU obtained from different solvents. The HRTEM suggests that these CDs are crystalline, which can be proven by the oriented lattice fringes in an enlarged view. (a)

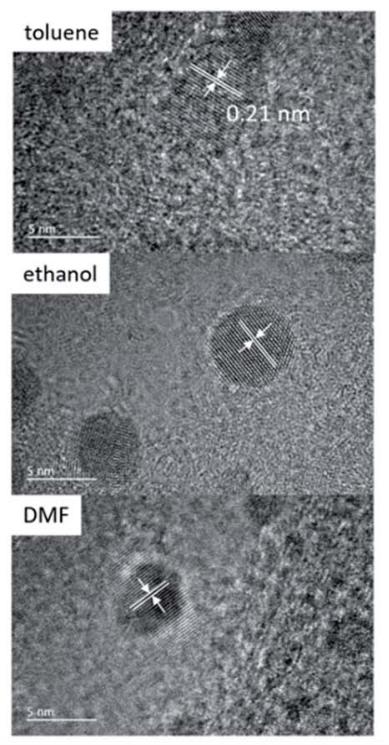

(b)
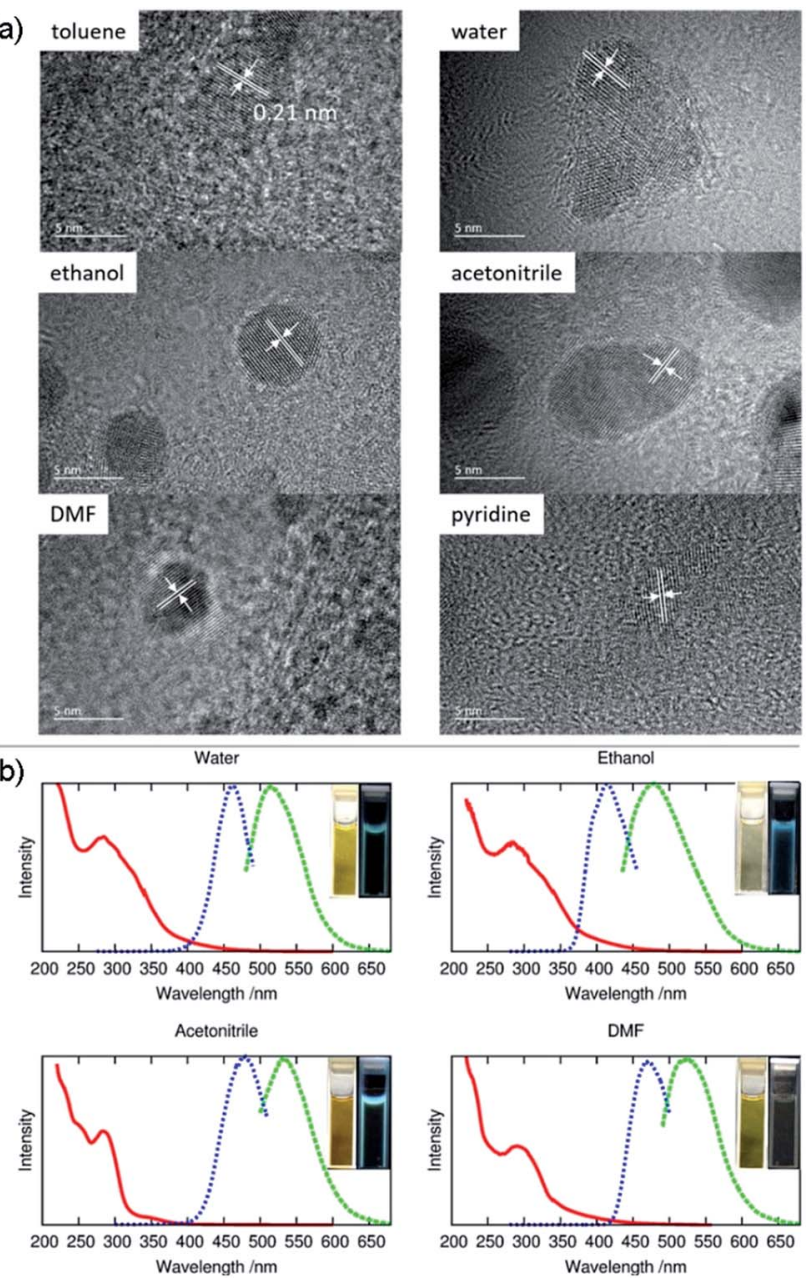

Fig. 3 Morphological characterization and optical studies: (a) high resolution transmission electron microscopy (HRTEM) images, (b) the normalized optical absorption spectra (red curve), photoluminescence excitation (blue curve) and photoluminescence emission (green curve) spectra of the carbon dots prepared by solvothermal methods with HATU in toluene, water, ethanol, acetonitrile, $\mathrm{N}, \mathrm{N}$-dimethylformamide (DMF) and pyridine, respectively.

The fringe separations are calculated to be $\sim 0.21 \mathrm{~nm}$ which are close to the (100) diffraction plane of graphite.

The energy dispersive X-ray spectroscopy (EDS) results (Fig. S6 and Table S2 $\dagger$ ) for ACD-HATU obtained from toluene show the peaks of the elements $\mathrm{C}, \mathrm{O}, \mathrm{N}, \mathrm{P}$ and $\mathrm{F}$. The ratio of $\mathrm{P}$ to $\mathrm{F}(1: 6)$ indicates the existence of $\mathrm{PF}_{6}{ }^{-}$, further suggesting an amphiphilic species from the structural aspect. The X-ray photoelectron spectroscopy (XPS) survey reveals that the sample consists mainly of carbon, nitrogen, oxygen and fluorine with a C 1s peak at $c a .287 \mathrm{eV}$, a N 1s peak at $c a .401 \mathrm{eV}$, an O 1s peak at $c a .533 \mathrm{eV}$ and a F 1s peak at $c a .691 \mathrm{eV}$. In detail, the C 1s spectrum shows two peaks at $285.7 \mathrm{eV}$ and $288.2 \mathrm{eV}$ which are attributed to $\mathrm{C}-\mathrm{C}$ and $\mathrm{C}-\mathrm{O}$, respectively. The $\mathrm{O} 1 \mathrm{~s}$ spectrum shows two peaks at $532.2 \mathrm{eV}$ and $534.0 \mathrm{eV}$, corresponding to C$\mathrm{OH}$ and $\mathrm{C}-\mathrm{O}-\mathrm{C}$, respectively (Fig. $\mathrm{S} 7 \dagger$ ).

The UV-vis spectra in Fig. 3b show absorption peaks around $290 \mathrm{~nm}$ and $350 \mathrm{~nm}$. The peaks around $290 \mathrm{~nm}$ indicate the 
presence of the $\mathrm{C}=\mathrm{C}$ bonds and the $\mathrm{C}=\mathrm{O}$ bonds, which further signify the formation of a CD core. The transition at $350 \mathrm{~nm}$ reflects the existence of the surface connected chemical groups. The corresponding PL spectra were recorded according to their excitation to display emissions around $470 \mathrm{~nm}, 510 \mathrm{~nm}$, $475 \mathrm{~nm}, 530 \mathrm{~nm}$ and $520 \mathrm{~nm}$ while in toluene, water, ethanol, acetonitrile and DMF, respectively. The emission of carbon dots normally undergoes a red shift upon an increase of solvent polarity according to previous reports. ${ }^{21}$ However, the PL in our case does not follow this trend. From the HRTEM images, we can see that the morphology of the samples obtained from DMF, water and acetonitrile were in an aggregated form with two or three original dots gathered together. The size of the single dots obtained from varied solvent conditions remains identical, only the solvent attachment on the surface alters the conjugation degree of the $\mathrm{sp}^{2}$ carbons $\mathrm{s}^{22}$ so as to result in different emission, although the fluorescence shifts are dominated mainly by the quantum confinement effect.

Even using water, we found that the solution still revealed an emission resolvable by the naked eye under the $365 \mathrm{~nm}$ excitation UV lamp. The fluorescence can be also proven from photoluminescence measurements (Fig. 3b). The relatively reduced emission can be attributed to an aggregation-caused quenching (ACQ) effect. ${ }^{23}$ In water, acetonitrile and pyridine, a few of the dots are aggregated together while in toluene, ethanol and DMF the dots are well-dispersed. Their relative quantum yields (quinine sulfate in $0.1 \mathrm{M} \mathrm{H}_{2} \mathrm{SO}_{4}$ as the reference) of the ACDs were $\sim 7 \%, \sim 2 \%, \sim 20 \%$ and $\sim 2 \%$ prepared from water, ethanol, acetonitrile and DMF, respectively. Anyway, the high-emissive characteristic of the ACDs can still ensure effective bio-imaging as demonstrated below. To give direct evidence for the amphiphilic property of the carbon dots, we also mixed the carbon dots prepared in toluene with deionized water, ultrasonicated them for two minutes and kept them for a couple of hours until the solution separated into two clear phases. Under the $365 \mathrm{~nm}$ UV lamp, both the lower aqueous and upper organic phases exhibited blue luminescence (Fig. S2c and S2d †) which means that a proportion of the carbon dots were transferred from the toluene to water sue to their amphiphilicity.

In addition to the QY, metal ionic doping also brought about improved crystallinity and monodispersity during the formation of the ACDs. A series of metal ions were tried for the doping during the synthesis of ACD-HATU, and the emissive property can generally be retained. The mother liquid of ACD-HATU after aluminum doping was centrifuged, and the deposit in the bottom was dropped onto a carbon membrane coated copper grid. This grid can be used on TEM-EDS for elemental determination. The mapping figures (Fig. S8†) showed the existence of aluminum. The doped sample exhibited a spherical shape, with diameters of 0.8-3.6 $\mathrm{nm}$ and well dispersed dots with a narrow size distribution as well as more concentrated dots in the same area, as compared with those from the non-doped materials (Fig. 4a and b). More interestingly, the doped ACDs show a larger Stokes shift $(\sim 100 \mathrm{~nm})$ than the undoped ones (Fig. S9†). Doping definitely minimized the self-quenching effect, leading to a longer PL lifetime of $6.7 \mathrm{~ns}$ (as compared (a)
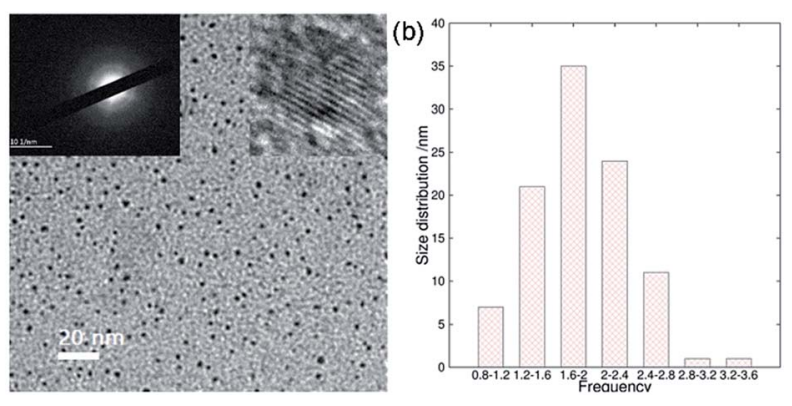

Fig. 4 Improved crystallinity and monodispersity due to metal ionic doping: (a) transmission electron microscopy (TEM) image with inset images of the selected area electron diffraction (SAED, left) and the high-resolution TEM image (right), and (b) the size distribution calculation based on one hundred dots of ACD-HATU doped with Al.

with the average lifetime of 2 ns for the undoped ACDs, see Fig. S10 in the ESI†), while maintaining a QY that is still high (ca. 20\%).

A narrow emission peak is usually one of the excellent PL properties in nanocrystals, ${ }^{24}$ however, CDs can display a unique excitation-dependent PL behavior. ${ }^{25}$ To further explore the fluorescence properties of our ACDs from this perspective, we carried out a detailed study varying the excitation wavelengths from 300 to $550 \mathrm{~nm}$ (see Fig. 5a and S11†). Upon excitation from $300 \mathrm{~nm}$ to $410 \mathrm{~nm}$, the maximum emission peak wavelength of the ACDs remained unchanged, only accompanied by a progressive increase in the emission intensity. Such a trend is in agreement with the maximum photoluminescence excitation at $410 \mathrm{~nm}$. Furthermore, as expected, the emission peak position underwent a bathochromic shift with a gradual decrease of intensity when the excitation wavelength was increased from $410 \mathrm{~nm}$ to $550 \mathrm{~nm}$. These results suggest that an excitationwavelength dependent PL characteristic can also be shown in our nanomaterials, allowing an exceptional multi-channel imaging effect as exemplified in the cellular studies below.

Although fluorescent probe techniques have been applied in bioimaging for years, demonstrations of the imaging of a single system under the control of detection channels are still scarce. ${ }^{26}$ The CDs we obtained are amphiphilic so that they are soluble directly in water and can be used as a biolabeling reagent. The mother liquid was freeze-dried, mixed with deionized water via ultrasonic treatment and centrifuged to separate the precipitate and water. The aqueous system containing ACD-HATU was then used for the cellular experiments. Fig. S12a $\uparrow$ shows the viability of the Hela cells using four parallel CCK-8 experiments. At concentrations up to $500 \mu \mathrm{g} \mathrm{mL} \mathrm{m}^{-1}$, the cell viability exceeds $85 \%$, whereas it exceeds $95 \%$ with a concentration of $100 \mu \mathrm{g} \mathrm{mL} \mathrm{L}^{-1}$. Such a progression suggests that the ACDs can be well endocytosed with low cytotoxicity. The cell spreading morphology is quite normal after incubation (see the bright field image in Fig. S12 $\dagger$ ), further indicating a favorable biocompatibility of the ACDs since this concentration and exposure time of the ACDs did not cause cell damage and apoptosis.

The effect of multi-channel imaging can be detected by confocal microscopes. As shown in Fig. 5b and S12, $\uparrow$ the cell 
(a)

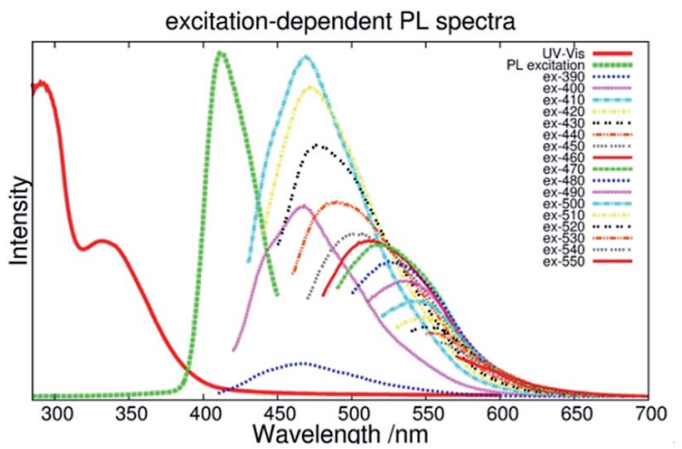

(b)

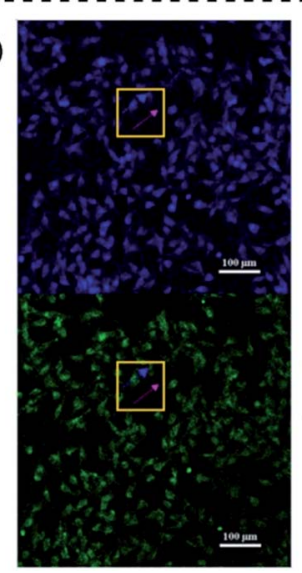

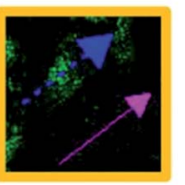

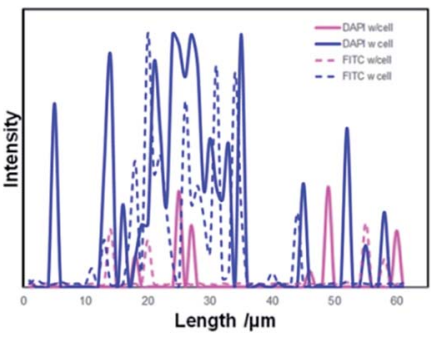

Fig. 5 Multi-channel cellular imaging studies. (a) UV-vis, photoluminescence excitation and emission spectra of ACD-HATU with increasing $\lambda_{\text {ex }}$ from $390 \mathrm{~nm}$ in $10 \mathrm{~nm}$ increments. (b) Selective dualchannel imaging: representative results of the confocal fluorescence microphotographs of Hela cells incubated with ACD-HATU, $\lambda_{\text {ex }}=$ $408 \mathrm{~nm}$ (blue, DAPI Channel) and $\lambda_{\mathrm{ex}}=488 \mathrm{~nm}$ (green, FITC Channel), and the brightness intensity readout curves along the arrows (the blue color and pink color show the paths going through a cell or not, respectively).

imaging using the ACDs can be clearly observed from both a DAPI channel under $408 \mathrm{~nm}$ excitation and an FITC channel under $488 \mathrm{~nm}$ excitation. The photoluminescence intensity along the cellular profile can also be quantified (see Fig. $5 \mathrm{~b}$ ), showing that the imaging works well from the distinct signal readouts. Meanwhile, the signal difference with and without going through a cell unambiguously signified the highresolution of the imaging when using these nanomaterials with high QYs. As we demonstrated above, the ACDs can undergo a remarkable excitation-dependent PL behavior. The investigation suggests that this performance can also occur at the cellular level, in agreement with the corresponding phenomena in solution. Such a spectrally tunable material might thus present a promising selective potential for advancing sensing or labeling at the nanoscale.

\section{Conclusions}

In summary, we have demonstrated a successful one-step solvothermal synthesis of high-emissive amphiphilic carbon dots from HATU, a typical coplanar compound, as the only precursor. A high luminescence quantum yield of the ACDs was observed, as well as a further improvement of crystallinity and monodispersity by metal ionic doping, in which the derivation of the rigid precursor structure, causing suppression of competitive energy loss, played a key role. Our materials, which are biocompatible with a low cytotoxicity, can also be applied in vivo to achieve high-resolution multi-channel cellular imaging, relying on an excitation-wavelength dependent emission behavior. We believe that the synthetic strategy demonstrated herein can be valuable for the development of next-generation light-emitting materials at the nanoscale for versatile applications with high brightness.

\section{Conflicts of interest}

There are no conflicts to declare.

\section{Acknowledgements}

This work was supported by the NSFC (21644005 and 21628401) and the National Program for Thousand Young Talents of China. H. A. and G. B. acknowledge the Carl Tryggers foundation (Grant No. CTS 16:536). G. B. also acknowledges the Ministry of Education and Science of Ukraine (project numbers 0115U003637 and 0117U001979).

\section{Notes and references}

1 X. Xu, R. Ray, Y. Gu, H. J. Ploehn, L. Gearheart, K. Raker and W. A. Scrivens, J. Am. Chem. Soc., 2004, 126, 12736.

2 (a) L. Cao, X. Wang, M. J. Meziani, F. Lu, H. Wang, P. G. Luo, Y. Lin, B. A. Harruff, L. M. Veca, D. Murray, S. Y. Xie and Y. P. Sun, J. Am. Chem. Soc., 2007, 129, 11318; (b) R. Liu, D. Wu, S. Liu, K. Koynov, W. Knoll and Q. Li, Angew. Chem., Int. Ed., 2009, 48, 4598; (c) P. Huang, J. Lin, X. S. Wang, Z. Wang, C. L. Zhang, M. He, K. Wang, F. Chen, Z. M. Li, G. X. Shen, D. X. Cui and X. Y. Chen, Adv. Mater., 2012, 24, 5104; (d) S. Sahu, B. Behera, T. K. Maiti and S. Mohapatra, Chem. Commun., 2012, 48, 8835 .

3 (a) S. Liu, J. Tian, L. Wang, Y. Zhang, X. Qin, Y. Luo, A. M. Asiri, A. O. Al-Youbi and X. Sun, Adv. Mater., 2012, 24, 2037; (b) W. Lu, X. Qin, S. Liu, G. Chang, Y. Zhang, Y. Luo, A. M. Asiri, A. O. Al-Youbi and X. Sun, Anal. Chem., 2012, 84, 5351; (c) F. R. Baptista, S. A. Belhout, S. Giordani and S. J. Quinn, Chem. Soc. Rev., 2015, 44, 4433.

4 (a) J. Tang, B. Kong, H. Wu, M. Xu, Y. C. Wang, Y. L. Wang, D. Y. Zhao and G. F. Zheng, Adv. Mater., 2013, 25, 6569; (b) M. Zheng, S. Liu, J. Li, D. Qu, H. F. Zhao, X. G. Guan, X. L. Hu, Z. G. Xie, X. B. Jing and Z. C. Sun, Adv. Mater., 2014, 26, 3554; (c) T. Feng, X. Ai, G. An, P. Yang and Y. Zhao, ACS Nano, 2016, 10, 4410.

5 (a) F. Wang, S. P. Pang, L. Wang, Q. Li, M. Kreiter and C. Y. Liu, Chem. Mater., 2010, 22, 4528; (b) S. Nandi, R. Malishev, K. P. Kootery, Y. Mirsky, S. Kolusheva and R. Jelinek, Chem. Commun., 2014, 50, 10299; (c) S. Nandi, M. Ritenberg and R. Jelinek, Analyst, 2015, 140, 4232; (d) 
J. Tan, J. Zhang, W. Li, L. Q. Zhang and D. M. Yue, J. Mater. Chem. C, 2016, 4, 10146.

6 (a) J. Wang, C. F. Wang and S. Chen, Angew. Chem., Int. Ed., 2012, 51, 9297; (b) O. Kozak, K. K. R. Datta, M. Greplova, V. Ranc, J. Kaslik and R. Zboril, J. Phys. Chem. C, 2013, 117, 24991; (c) Y. Zhou, S. Sharma, Z. Peng and R. Leblanc, Polymers, 2017, 9, 67.

7 (a) B. G. Wang, A. X. Song, L. Feng, H. Ruan, H. G. Li, S. L. Dong and J. C. Hao, ACS Appl. Mater. Interfaces, 2015, 7, 6919; (b) D. P. Kong, F. Y. Yan, Y. M. Luo, Q. H. Ye, S. Y. S. Zhou and L. Chen, Anal. Chim. Acta, 2017, 953, 63; (c) X. Liu, Y. Gao, X. Wang, S. Wu and Z. Tang, J. Nanosci. Nanotechnol., 2011, 11, 1941; (d) S. L. Zhao, H. J. Yin, L. Du, L. C. He, K. Zhao, L. Chang, G. P. Yin, H. J. Zhao, S. Q. Liu and Z. Y. Tang, ACS Nano, 2014, 8, 12660.

8 (a) Q.-C. Wang, D.-H. Qu, J. Ren, K. Chen and H. Tian, Angew. Chem., Int. Ed., 2004, 116, 2715; (b) H. Deng, M. A. Olson, J. F. Stoddart and O. M. Yaghi, Nat. Chem., 2010, 2, 439; (c) Y. Hong, J. W. Lam and B. Z. Tang, Chem. Soc. Rev., 2011, 40, 5361; (d) R. Y. Sun and L. L. Zhu, RSC Adv., 2016, 6, 73527; (e) Y. Zhou, M. Yang, K. Sun, Z. Tang and N. A. Kotov, J. Am. Chem. Soc., 2010, 132, 6006; (f) Y. Zhou, Z. Zhu, W. Huang, W. Liu, S. Wu, X. Liu, Y. Gao, W. Zhang and Z. Tang, Angew. Chem., Int. Ed., 2011, 50, 11456.

9 (a) X. H. Gao and S. M. Nie, J. Phys. Chem. B, 2003, 107, 11575; (b) D. Mocatta, G. Cohen, J. Schattner, O. Millo, E. Rabani and U. Banin, Science, 2011, 332, 77; (c) J. H. Choi, A. T. Fafarman, S. J. Oh, D. K. Ko, D. K. Kim, B. T. Diroll, S. Muramoto, J. G. Gillen, C. B. Murray and C. R. Kagan, Nano Lett., 2012, 12, 2631; (d) X. Li, S. P. Lau, L. Tang, R. Ji and P. Yang, Nanoscale, 2014, 6, 5323; (e) F. Qian, X. Li, L. Tang, S. K. Lai, C. Lu and S. P. Lau, AIP Adv., 2016, 6, 075116.

10 (a) X. Y. Li, H. Q. Wang, Y. Shimizu, A. Pyatenko, K. Kawaguchi and N. Koshizaki, Chem. Commun., 2011, 47, 932; (b) H. Ming, Z. Ma, Y. Liu, K. M. Pan, H. Yu, F. Wang and Z. H. Kang, Dalton Trans., 2012, 41, 9526; (c) J. Kim and J. S. Suh, ACS Nano, 2014, 8, 4190; (d) Y. Liang, C. Lu, D. Ding, M. Zhao, D. Wang, C. Hu, J. Qiu, G. Xie and Z. Tang, Chem. Sci., 2015, 6, 4103.

11 (a) B. C. M. Martindale, G. A. M. Hutton, C. A. Caputo and E. Reisner, J. Am. Chem. Soc., 2015, 137, 6018; (b) A. M. Schwenke, S. Hoeppener and U. S. Schubert, Adv. Mater., 2015, 27, 4113; (c) M. Zheng, S. B. Ruan, S. Liu, T. T. Sun, D. Qu, H. F. Zhao, Z. G. Xie, H. L. Gao, X. B. Jing and Z. C. Sun, ACS Nano, 2015, 9, 11455; (d) B. Wang, H.-J. Liu and Y. Chen, RSC Adv., 2016, 6, 2141; (e) F. Yuan, Z. Wang, X. Li, Y. Li, Z. Tan, L. Fan and S. Yang, Adv. Mater., 2017, 29, 1604436; (f) L. Tang, R. Ji, X. Cao, J. Lin, H. Jiang, X. Li, K. S. Teng, C. M. Luk, S. Zeng, J. Hao and S. P. Lau, ACS Nano, 2012, 6, 5102.

12 L. Porres, A. Holland, L. O. Palsson, A. P. Monkman, C. Kemp and A. Beeby, J. Fluoresc., 2006, 16, 267.

13 A. T. R. Williams, S. A. Winfield and J. N. Miller, Analyst, 1983, 108, 1067.

14 (a) A. D. Becke, Phys. Rev. A: At., Mol., Opt. Phys., 1988, 38, 3098; (b) C. Lee, W. Yang and R. G. Parr, Phys. Rev. B: Condens. Matter Mater. Phys., 1988, 37, 785.
15 (a) R. Krishnan, J. S. Binkley, R. Seeger and J. A. Pople, J. Chem. Phys., 1980, 72, 650; (b) M. J. Frisch, J. A. Pople and J. S. Binkley, J. Chem. Phys., 1984, 80, 3265.

16 M. J. Frisch, G. W. Trucks, H. B. Schlegel, G. E. Scuseria, M. A. Robb, J. R. Cheeseman, G. Scalmani, V. Barone, G. A. Petersson, H. Nakatsuji, X. Li, M. Caricato, A. V. Marenich, J. Bloino, B. G. Janesko, R. Gomperts, B. Mennucci, H. P. Hratchian, J. V. Ortiz, A. F. Izmaylov, J. L. Sonnenberg, D. Williams-Young, F. Ding, F. Lipparini, F. Egidi, J. Goings, B. Peng, A. Petrone, T. Henderson, D. Ranasinghe, V. G. Zakrzewski, J. Gao, N. Rega, G. Zheng, W. Liang, M. Hada, M. Ehara, K. Toyota, R. Fukuda, J. Hasegawa, M. Ishida, T. Nakajima, Y. Honda, O. Kitao, H. Nakai, T. Vreven, K. Throssell, J. A. Montgomery Jr, J. E. Peralta, F. Ogliaro, M. J. Bearpark, J. J. Heyd, E. N. Brothers, K. N. Kudin, V. N. Staroverov, T. A. Keith, R. Kobayashi, J. Normand, K. Raghavachari, A. P. Rendell, J. C. Burant, S. S. Iyengar, J. Tomasi, M. Cossi, J. M. Millam, M. Klene, C. Adamo, R. Cammi, J. W. Ochterski, R. L. Martin, K. Morokuma, O. Farkas, J. B. Foresman and D. J. Fox, Gaussian 16, Revision A.03, Gaussian, Inc., Wallingford CT, 2016.

17 R. F. W. Bader, Atoms in Molecules. A Quantum Theory, Clarendon Press, Oxford, 1990.

18 T. A. Keith, AIMAll (Version 10.07.25), TK Gristmill Soft-ware, Overland Park KS, USA, 2010.

19 V. Georgakilas, J. A. Perman, J. Tucek and R. Zboril, Chem. Rev., 2015, 115, 4744.

20 A. T. Onawole, A. F. Al-Ahmadi, Y. S. Mary, C. Y. Panicker, N. Ullah, S. Armaković, S. J. Armaković, C. Van Alsenoy and A. A. Al-Saadi, J. Mol. Struct., 2017, 1147, 266.

21 S. Paulo, G. Stoica, W. Cambarau, E. Martinez-Ferrero and E. Palomares, Synth. Met., 2016, 222, 17.

22 S. Zhu, Y. Song, X. Zhao, J. Shao, J. Zhang and B. Yang, Nano Res., 2015, 8, 355.

23 S. W. Thomas, G. D. Joly and T. M. Swager, Chem. Rev., 2007, 107, 1339.

24 (a) V. I. Klimov, A. A. Mikhailovsky, S. Xu, A. Malko, J. A. Hollingsworth, C. A. Leatherdale, H. J. Eisler and M. G. Bawendi, Science, 2000, 290, 314; (b) X. G. Peng, L. Manna, W. D. Yang, J. Wickham, E. Scher, A. Kadavanich and A. P. Alivisatos, Nature, 2000, 404, 59; (c) M. J. Bowers, J. R. McBride and S. J. Rosenthal, J. Am. Chem. Soc., 2005, 127, 15378; (d) C. Y. Ang, L. Giam, Z. M. Chan, A. W. H. Lin, H. Gu, E. Devlin, G. C. Papoefthymiou, S. T. Selvan and J. Y. Ying, Adv. Mater., 2009, 21, 869; (e) L. L. Zhu, C. Y. Ang, X. Li, K. T. Nguyen, S. Y. Tan, H. Agren and Y. L. Zhao, Adv. Mater., 2012, 24, 4020.

25 Y. P. Sun, B. Zhou, Y. Lin, W. Wang, K. A. S. Fernando, P. Pathak, M. J. Meziani, B. A. Harruff, X. Wang, H. F. Wang, P. J. G. Luo, H. Yang, M. E. Kose, B. L. Chen, L. M. Veca and S. Y. Xie, J. Am. Chem. Soc., 2006, 128, 7756. 26 (a) L. L. Zhu, X. Li, Q. Zhang, X. Ma, M. H. Li, H. C. Zhang, Z. Luo, H. Agren and Y. L. Zhao, J. Am. Chem. Soc., 2013, 135, 5175; (b) Y. Y. Zhou, Y. P. Zhuang, X. Li, H. Agren, L. Yu, J. D. Ding and L. L. Zhu, Chem.-Eur. J., 2017, 23, 7642. 